\title{
VOLUME CHANGES OF WHOLE BRAIN GRAY AND WHITE MATTER IN CHILDREN PATIENTS WITH TOURETTE'S SYNDROME: EVIDENCE FROM VOXEL-BASED MORPHOMETRY
}

\author{
Y. Liu ${ }^{1}$, Y. Peng ${ }^{1}$, P. Gao ${ }^{2}$, B. $\mathrm{Nie}^{3}$ \\ ${ }^{I}$ Radiology Department, Beijing Children's Hospital Affiliated to Capital Medical University, \\ ${ }^{2}$ Neuroradiology Department, Beijing Tian Tan Hospital Affiliated to Capital Medical University, ${ }^{3}$ Institute \\ of High Energy Physics, Chinese Academy of Science, Beijing, China
}

Background and aims: Tourette syndrome (TS) is a disorder of chronic motor and vocal tics that begins in childhood and fluctuate in severity across time. To identify TS related abnormalities in brain, we used optimized voxel-based morphometry (VBM) and provide a quantitative measure of volumes.

Methods: Three dimensional T1-weighted MRI were acquired in $31 \mathrm{TS}$ children(28 boys, 3 girls, mean age $=8.4$ years, range 2-15 years) and 50 age-matched controls on a 1.5 Tesla Philips scanner. Images were analyzed using a version of VBM2 in SPM2.

Results: Using VBM, TS patients showed significant increases in gray matter volumes in left superior parietal lobule and left parahippocampal gyrus. Decreases in gray matter volumes were seen in brain stem. Decrease in white matter volume was found in left inferior occipital lobe. Increase in white matter volume was detected in pons $(\mathrm{P}<0.05)$. Tic severity was no correlated with any volume changes of gray and white matter in brain. Tic course was negatively correlated with the gray volume of left para-hippocampal gyrus $(\mathrm{P}=0.045)$.

Conclusions: Our MRI findings suggest that abnormalities of parietal-temporal-occipital association regions and brain stem are associated with TS. Detection in changes of gray and white volumes may be helpful for the early diagnosis and evaluation of TS. 